\title{
Analysis of kinematic, kinetic and electromyographic patterns during root canal preparation with rotary and manual instruments
}

\author{
Braulio PASTERNAK-JÚNIOR', Manoel Damião de SOUSA NETO², Valdeci Carlos DIONíSIO³ , Jesus Djalma PÉCORA², \\ Ricardo Gariba SILVA ${ }^{1}$ \\ 1- DDS, MSc, PhD, Department of Endodontics, Dental School, University of Ribeirão Preto - UNAERP, Ribeirão Preto, SP, Brazil.
2- DDS, MSc, PhD, Department of Restorative Dentistry, Dental School of Ribeirão Preto, University of São Paulo-USP, Ribeirão Preto, SP, Brazil. \\ 3- DDS, MSc, PhD, Department of Kinesiology, University of Ribeirão Preto - UNAERP, Ribeirão Preto, SP, Brazil.
}

Corresponding address: Prof. Dr. Manoel D. Sousa Neto - Rua Célia de Oliveira Meirelles, 350 - Jardim Canadá - 14024070 - Ribeirão Preto - SP - Brasil

- Phone: +55-16-623-6002 - Fax: +55-16-3603-6783 - e-mail: sousanet@forp.usp.com

Received: November 13, 2010 - Modification: August 26, 2010 - Accepted: October 26, 2010

\section{ABSTRACT}

$\mathrm{O}$ bjective: This study assessed the muscular activity during root canal preparation through kinematics, kinetics, and electromyography (EMG). Material and Methods: The operators prepared one canal with RaCe rotary instruments and another with Flexofiles. The kinematics of the major joints was reconstructed using an optoelectronic system and electromyographic responses of the flexor carpi radialis, extensor carpi radialis, brachioradialis, biceps brachii, triceps brachii, middle deltoid, and upper trapezius were recorded. The joint torques of the shoulder, elbow and wrist were calculated using inverse dynamics. In the kinematic analysis, angular movements of the wrist and elbow were classified as low risk factors for work-related musculoskeletal disorders. With respect to the shoulder, the classification was medium-risk. Results: There was no significant difference revealed by the kinetic reports. The EMG results showed that for the middle deltoid and upper trapezius the rotary instrumentation elicited higher values. The flexor carpi radialis and extensor carpi radialis, as well as the brachioradialis showed a higher value with the manual method. Conclusion: The muscular recruitment for accomplishment of articular movements for root canal preparation with either the rotary or manual techniques is distinct. Nevertheless, the rotary instrument presented less difficulty in the generation of the joint torque in each articulation, thus, presenting a greater uniformity of joint torques.

Key words: Kinematics. Kinetics. Electromyography. Endodontics. Dental pulp cavity.

\section{INTRODUCTION}

The purpose of ergonomics applied to Dentistry is to obtain means and systems to decrease physical and mental stress, as well as prevent diseases related to dental practices ${ }^{6}$. Work-related musculoskeletal disorders (WMSD) have increased in recent decades and are factors that commonly lead to professionals being unable to work, with financial and medical consequences ${ }^{3}$. In the case of dentists, incorrect posture is a cause of cervical column, neck, and shoulder disorders, besides hand neuropathies, due to the continuous use of dental instruments and tools ${ }^{1,23}$. Alexopoulos, Stathi and Charizani ${ }^{2}$ (2004) observed that 52\% of the Greek dentists who participated in a related study, reported at least one osteomuscular disorder. Adapting working positions to ergonomic rules is one of the measures to prevent WMSD, which can significantly contribute to a reduction in physical effort, thereby bringing benefits to the daily routine of professional activities $8,9,18$.

Another consideration is equipment design which is extremely important in terms of decreasing muscular fatigue and, consequently, reducing physical and mental stress, as highlighted by Parsell, et al. ${ }^{16}$ (2000). Posture-comfort stools with arm supports can offer musculoskeletal benefits when cavity preparations are carried out by dentists. Simmer-Beck, et al. ${ }^{22}$ (2006) reported 
that it is possible to reduce WMSD with ergonomic adaptations to the clinical mirrors. Morse, et al.13 (2003) also reported an association between an increase in the hours of use the ultrasonic scaler, the instrument of choice for calculus debridement, and an increase in tendonitis in the upper extremities resulting from dental hygiene procedures.

Régis-Filho ${ }^{18}$ (1997) demonstrated, by the muscular biomechanics expressed by kinematic registrations (working angles), kinetic (muscular torque) and electromyographic (neuromuscular activity graphically represented by the electrical activity of the muscle), that endodontics, and more specifically root canal preparation, is a dental practice that commonly causes WMSD, surpassing the clinical procedures of extractions, scaling and curettage, as well as amalgam condensation.

Ozawa, et al. ${ }^{14}$ (1998) reported that of a group of 192 dentists $79.2 \%$ had fatigue and pain in the hands and forearms, which occurred with greater intensity during or after root canal preparation. With the aim of reducing these symptoms, endodontic instrument handles manufactured according to ergonomic principles have been proposed, since larger diameters facilitate the gripping of the instruments and reduce muscular electromyographic activity ${ }^{15}$.

As in the case of several other medical and dental specializations, endodontics has evolved in recent years, setting forward greater precision, reduced possibility of mistakes and injuries, less discomfort to the patients and faster procedures ${ }^{27}$. Some authors have suggested that, in addition to these factors, root canal preparation with nickeltitanium (NiTi) instruments is associated with the least stress and operating fatigue, especially due to the decrease in working time when compared with manual instrumentation with stainless steel. The development of endodontic instruments made from $\mathrm{NiTi}$ alloy has the potential to enhance the shaping of narrow, curved canals, a procedure which has proved difficult to achieve using stainless steels instruments because of their inherent stiffness $7,17,21,24$.

The objective of this study was to verify, in vivo, the results obtained from the kinematic kinetics, and electromyographic records in endodontists during the preparation of simulated root canals using rotary and manual instrumentation techniques.

\section{MATERIAL AND METHODS}

Eight right-handed healthy subjects, four females and four males, between 23 and 39 years of age, participated in this study. All of them were endodontists with clinical experience accumulated over at least 2 years.

Each endodontist, seated on a dental stool in the "12 o'clock" position, prepared two simulated canals with $20 \mathrm{~mm}$ in resin blocks with diameters corresponding to an instrument size 20 and curvatures of $40^{\circ}$ (Crinodonto Produtos Odontológicos, Curitiba, PR, Brazil). RaCe 25.02 rotary $\mathrm{Ni}-\mathrm{Ti}$ (FKG, La Chaux-of-Fonds, Orbe, Switzerland) and Flexo-file 25.02 (DentsplyMaillefer, Ballaigues, Orbe, Switzerland) instruments were used. Prior to the use of the above-described instruments, the resin blocks were attached to a lathe machine and prepared. In the cervical third, the preparation was carried out with rotary PreRaCe instruments 40.10 and 35.08 (FKG, Reads Chaux-de-Fonds, Orbe, Switzerland), following the crown-down instrumentation procedure, where the dentist essentially works from the crown of the tooth, shaping the canal towards the apex. The instruments are used in a large to small sequence. Flexo-files 40.02, 35.02 and 30.02 were used by the manual group, RaCe 25.06 and 25.04 instruments by the rotary group. The steps for instrumentation in groups manual and rotary are shown in Figure 1. The purpose was to reach the working length $(\mathrm{WL}=20 \mathrm{~mm}$ ) with the 25.02 instrument. Once the WL was reached, 10 repetitions of 3 seesaw movements each were carried out while data was collected on muscular activity over 4-s periods. The reading of the movements would be very extensive if it were done throughout the whole preparation procedure, using all the instruments, thence, only the last instrument employed for each preparation was used (25.02, which correspond to $0.25 \mathrm{~mm}$ diameter in the initial tip with $0.02 \mathrm{~mm}$ taper at each millimeter in the active part of the instrument). That was true for the manual method, where the instrument is handled with fingers tips, as well as for the rotating instrument. In the rotary method, the instruments, which are driven by electric or pneumatic motors, make a continuous $360^{\circ}$ rotation.

Electromyographic (EMG) data were collected using surface electrodes attached to the skin over the muscle of interest. The skin was cleaned to adherence of the electrodes and detection of the EMG. Bipolar surface electrodes (model 2.2L

\begin{tabular}{|c|c|c|c|}
\hline Step & $\begin{array}{c}\text { Crown-down } \\
\text { instrumentation (mm) }\end{array}$ & Manual & Rotary \\
\hline 1 & 12 & $40 / .10$ & $40 / .10$ \\
\hline 2 & 14 & $35 / .08$ & $35 / .08$ \\
\hline 3 & 16 & $40 / .02$ & $25 / .06$ \\
\hline 4 & 18 & $35 / .02$ & $25 / .04$ \\
\hline 5 & 20 (Working Length) & $30 / .02$ & $25 / .02$ \\
\hline 6 & 20 (Working Length) & 25.02 & \\
\hline
\end{tabular}

Figure 1- Instrumentation steps for manual and rotary methods 
Delsys Inc., Boston, MA, USA) were attached over the flexor carpi radialis, extensor carpi radialis, brachioradialis, biceps brachii, triceps brachii, middle deltoid, and upper trapezius. The electrodes were positioned in parallel with the muscular fibers, at approximately two centimeters from each muscle motor point (between the motor point and the tendon). This location was determined after an isometric contraction of each muscle group. The EMG signals were amplified (x2000), bandpass filtered $(20-450 \mathrm{~Hz})$ and recorded. The data were digitized at 12 bits and collected by an IBM computer at $1000 \mathrm{~Hz}$.

For the analysis of the movements, $X, Y$, and $Z$ coordinates were recorded utilizing LED (Light Emission Diode) markers fastened to the temporomandibular joint, to the hip (over the iliac crest), shoulder (lateral portion of acromion), elbow (lateral epicondyle), wrist (Lister's tubercle), and hand (metacarpal head). The infrared signal emission of these markers was captured at a frequency of $100 \mathrm{~Hz}$, by a three-dimensional OPTOTRAK 3020 optical system (Digital Northern Inc., Waterloo, Ontario, Canada).

\section{Data processing}

The resulting electromyographic EMG signals in millivolts ( $\mathrm{mV}$ ), and the $X, Y$, and $Z$ positional data were synchronized by an ODAU II - Optotrak Data Acquisition Unit II, and mathematically treated using MatLab Code (Math Works Inc., version 6.0).

The EMG signals were rectified, filtered (lowpass at $20 \mathrm{~Hz}$ using a second-order Butterworth filter), and normalized by the maximum voluntary isometric contraction (MVIC) of each subject. The averaged EMG of the MVIC was calculated within the 500-1000 milliseconds (ms) interval from the beginning an isometric contraction. For all MVIC tests, the positions were based on the Kendall, et al. ${ }^{9}$ (2005).
The linear displacement of the markers, the joint torque generated by the shoulder, elbow, and wrist, as well as deviations, angular velocity, and accelerations, were also calculated. The anthropometric data, center of mass, and moment of inertia of the segments were calculated based on the weight and sex of the subject, according to Zatsiorsky's model, modified by De Leva ${ }^{5}$ (1996). Based on these data, the joint torque of the shoulder, elbow, and wrist were calculated through inverse dynamics ${ }^{19,20}$ and later normalized to each subject's body weight.

The joint torque averages $(\mathrm{Nm} / \mathrm{kg})$ for the shoulder, elbow, and wrist, as well as the EMG signal normalized values were calculated at intervals of $100 \mathrm{~ms}$ each, starting from the beginning of the movement of the wrist assessed by measuring the angular displacement.

\section{Data analysis}

The Student t-test for paired samples was applied according to the experimental design and type of variable, considering that this study involved a parametric variable and two experimental groups.

For this analysis, the average of the angular displacement, joint torques and EMG across all subjects during the movement was calculated across intervals of 100 ms, for each instrumentation method. After, the methods were compared through the paired test, because the same individuals carried out the instrumentation with both methods. A significance level of $5 \%$ was established.

\section{RESULTS}

\section{Kinematics}

The results of the angular displacements are shown in Table 1. It was ascertained that there were differences between the results obtained with the use of manual and rotary techniques with regard

Table 1- Distribution of the sample based on maximum (max), minimum (min) angular displacement and angular excursion $(A E)$ between the articulations over all repetitions across all subjects regarding the direction of joint movement. Average values expressed in degrees and standard deviations. This angle are of the flexion/extension for shoulder and elbow (external angle), and radial/ulnar deviation for wrist

\begin{tabular}{ccccc}
\hline Variable & $\begin{array}{c}\text { Manual Method } \\
(\mathbf{n = 8})\end{array}$ & $\begin{array}{c}\text { Rotary Method } \\
(\mathbf{n = 8})\end{array}$ & $\begin{array}{c}\text { Difference between the } \\
\text { averages (SD) }\end{array}$ & $\mathbf{p}$ \\
\hline Max_shoulder & $33.55(13.25)$ & $38.49(14.23)$ & $4.94(4.31)$ & 0.01 \\
\hline AE_shoulder & $0.49(0.29)$ & $0.23(0.10)$ & $0.26(0.25)$ & 0.02 \\
\hline Min_elbow & $64.53(9.78)$ & $64.02(11.24)$ & $0.50(9.61)$ & 0.89 \\
Max_elbow & $65.49(9.93)$ & $64.57(11.05)$ & $0.92(9.83)$ & 0.80 \\
\hline AE_elbow & $0.96(0.65)$ & $0.55(0.33)$ & $0.41(0.56)$ & 0.07 \\
\hline Min_wrist & $11.09(5.94)$ & $17.37(10.21)$ & $6.28(6.13)$ & 0.02 \\
\hline Max_wrist & $8.92(4.73)$ & $15.20(11.02)$ & $6.27(6.93)$ & 0.04 \\
AE_wrist & $2.16(1.78)$ & $2.175(1.76)$ & $0.011(2.981)$ & 0.99 \\
\hline
\end{tabular}


to the variables of the minimum (min.), maximum (max.), and angular extension (AE) techniques for the shoulder, and low and maximum techniques for the wrist.

\section{Kinetics}

The average measured joint torque values for the shoulder, elbow, and wrist $(\mathrm{Nm} / \mathrm{kg})$ are given in
Table 2. It can be observed that the instrumentation method did not influence any of the analyzed variables in a statistically significant way. The use of the rotary technique led to a higher uniformity of the results obtained regarding the torque values during the time intervals. The manual technique showed high oscillations during the time intervals analyzed (Figure 2).

Table 2- Joint torque values normalized to each subject's body weight for the shoulder, elbow, and wrist (Nm/kg) during the moviments for the preparation of simulated root canals with manual and rotary instruments. Average values expressed in $\mathrm{Nm} / \mathrm{kg}$ and standard deviation. The positive and negative values represent the clockwise and counterclockwise directions respectively

\begin{tabular}{ccccc}
\hline Variable & $\begin{array}{c}\text { Manual Method } \\
(\mathbf{n}=\mathbf{8})\end{array}$ & $\begin{array}{c}\text { Rotary Method } \\
(\mathbf{n}=\mathbf{8})\end{array}$ & $\begin{array}{c}\text { Difference between the } \\
\text { averages (SD) }\end{array}$ & $\mathbf{p}$ \\
\hline Shoulder & $-0.0035(0.1886)$ & $-0.0067(0.0579)$ & $0.00317(0.22037)$ & 0.941 \\
Elbow & $0.0009(0.1004)$ & $-0.0026(0.0361)$ & $0.00348(0.11869)$ & 0.880 \\
Wrist & $0.0029(0.1016)$ & $-0.0010(0.0356)$ & $0.00394(0.10902)$ & 0.853 \\
\hline
\end{tabular}

A

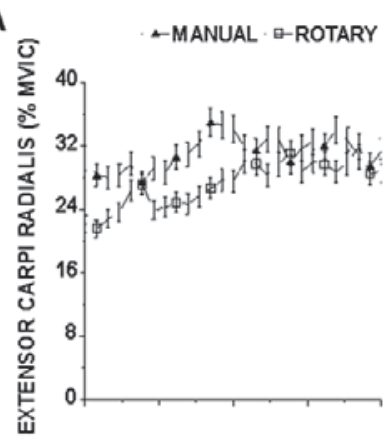

C

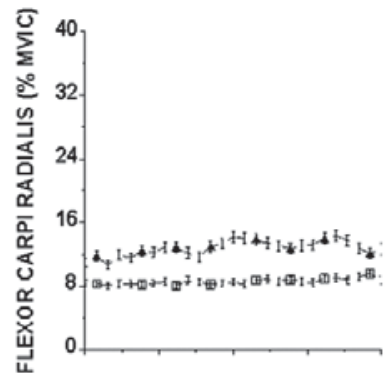

E

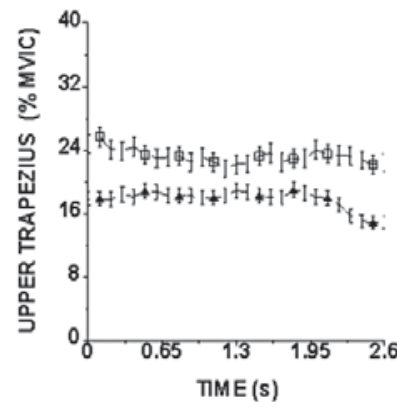

B

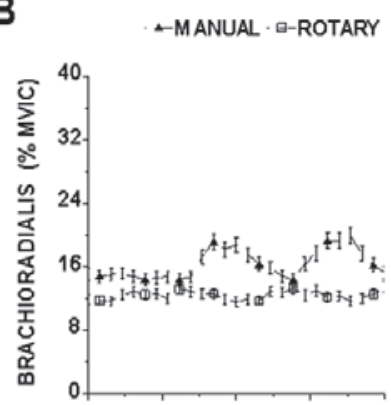

D

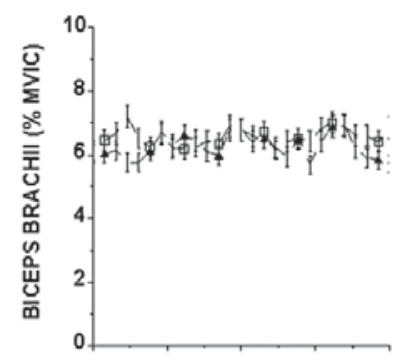

F

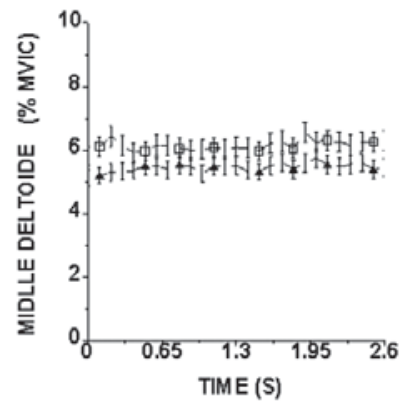

Figure 2-Average and standard error across all subjects of the normalized electromyography (EMG) activity of the extensor carpi radialis $(A)$, brachioradialis $(B)$, flexor carpi radialis $(C)$, biceps brachii $(D)$, upper trapezius $(E)$ and middle deltoid $(F)$ during all intervals calculated for each instrumental method 
A

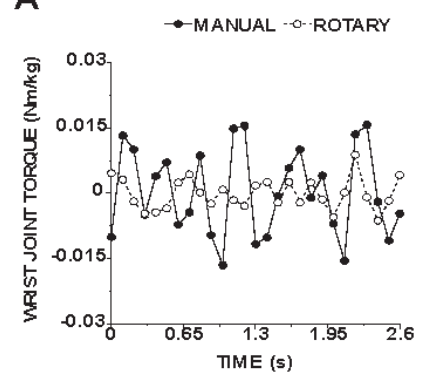

B

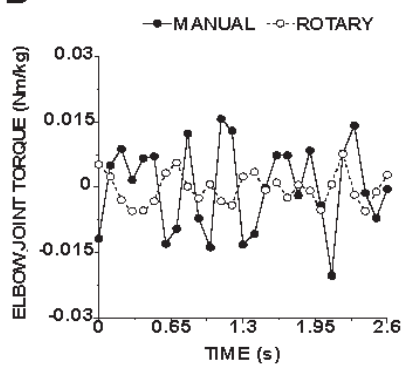

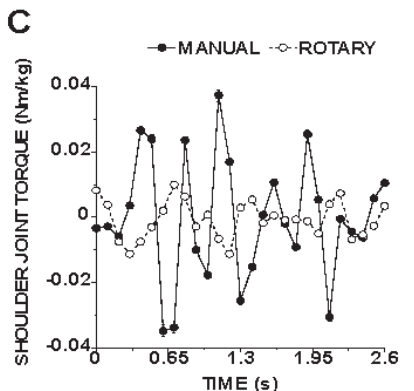

Figure 3- Comparison between the manual and rotary methods according to the average and standard error across all subjects of the joint torque $(\mathrm{Nm} / \mathrm{kg}$ ) for the wrist $(A)$, elbow $(B)$ and shoulder $(C)$, during all intervals calculated (each 100 $\mathrm{ms}$ ) for each instrumental method

\begin{tabular}{|l|l|l|l|}
\hline Limit risk & Low risk & Medium risk & High risk \\
\hline Movement & & & \\
\hline Wrist Extension & 0 to $15^{\circ}$ & $>15$ a $45^{\circ}$ & $>45^{\circ}$ \\
\hline Wrist Ulnar Deviation & 0 to $20^{\circ}$ & $>20$ a $30^{\circ}$ & $>31^{\circ}$ \\
\hline Wrist Radial Deviation & 0 to $10^{\circ}$ & $>10$ a $20^{\circ}$ & $>20^{\circ}$ \\
\hline Elbow Flexion & 0 to $90^{\circ}$ & $>90$ a $120^{\circ}$ & $>120^{\circ}$ \\
\hline Shoulder Flexion & 0 to $30^{\circ}$ & $>30$ a $60^{\circ}$ & $>60^{\circ}$ \\
\hline Shoulder Abduction & 0 to $30^{\circ}$ & $>30$ a $60^{\circ}$ & $>60^{\circ}$ \\
\hline Shoulder Half Rotation & 0 to $10^{\circ}$ & $>10$ a $45^{\circ}$ & $>45^{\circ}$ \\
\hline Head Flexion & 0 to $10^{\circ}$ & $>10$ a $30^{\circ}$ & $>30^{\circ}$ \\
\hline Wrist Flexion & 0 to $15^{\circ}$ & $>15$ a $45^{\circ}$ & $>45^{\circ}$ \\
\hline
\end{tabular}

Figure 4- Angular movement of the articulation limit before the appearance of work-related musculoskeletal disorders (WMSD) classified as low, medium or high risks by Bramson ${ }^{4}(1998)$

\section{Electromyography}

Normalized EMG values expressed as a percentage of MVIC are given in Table 3.

It was observed for the manual method that there was a higher EMG activity in the extensor carpi radialis, flexor carpi radialis and brachioradialis muscles, and lower EMG activity in the middle deltoid and upper trapezius compared with the rotary method. The biceps brachii and triceps brachii did not show statistically significant differences in relation to the preparation methods (Figure 3 ).

\section{DISCUSSION}

With the advent of rotary instruments, general dentists and endodontists can carry out treatments faster and with a better final quality of the preparations ${ }^{26}$. Rotary instruments, however, should allow less physical effort and a lower probability of injury for dental professionals. However, no study has ever compared rotary and manual techniques with regard to the muscular and biomechanical aspects of these devices.

Bramson, Smith and Romagnoli ${ }^{4}$ (1998) verified that the repetitive movement of an articulation has a limit before the appearance of WMSD and that depending on the extent to which this limit is exceeded the risk of WMSD is classified as low, medium or high (Figure 4). Through the comparative kinematic analysis performed in this study, the angle displacements for the wrist and elbow verified during the preparations using rotary or manual instruments were classified as low risk. As for the shoulder, these movements were classified as medium risk.

The joint torque represents the muscular action on the articulation added to the passive elements of which it is composed, such as ligaments, tendons, and articulation capsules ${ }^{20}$. Through the results obtained, we can observe that, despite the lack of a statistically significant difference between the root canal preparation techniques, the quality analysis of the behavior of the shoulder, elbow, and wrist joint torques revealed a greater uniformity of values during the intervals for the rotary instrumentation, allowing for a more homogeneous effort without high peaks. On the other hand, the analysis revealed many oscillations in joint torque behavior for the manual instruments in the three articulations, thus suggesting the need for recruitment of a greater number of muscles to generate such torques and execute the task. 
It can be observed, through the EMG results, that there was a difference between the instrumentation techniques for most of the muscular groups under analysis. Except for the middle deltoid and trapezius, where use of rotary instrumentation resulted in a higher EMG level, the majority of the muscular groups required less effort when using this technique. The explanation for the occurrence of a higher EMG activity during use of the manual instrumentation lies in the very fact that there is a need greater apprehend of instruments to execute the task. The wrist and the elbow position are fundamental for this execution. During use of rotary instruments, there is the need to hold the equipment, but less effort is required to penetrate and work inside the root canals. In this case the professionals employ a different motor strategy, using the muscles closer to the shoulder.

It is important to emphasize that the continuous activity of both the extensor carpi radialis and flexor carpi radialis observed in this study with the manual technique, as well as of the brachioradialis muscle, upper trapezius and middle deltoid with the rotary instrumentation, characterize a static contraction. The tension generated by the static contraction may contribute to the alteration of microcirculation in the muscle ${ }^{10}$, thus determining the appearance of painful points called "trigger points", characteristic of myofascial pain ${ }^{25,26}$. Myofascial pain causes more muscular tension, which can lead to poor blood circulation, which yields more pain, thence generating a vicious cycle. Myofascial pain affects various regions of the body, but one of the most common points is the trapezius muscle region, which is important for the head posture positioning, as well as for the execution of the scapulae function.

The muscular recruitment for performing articular movements for root canal preparation with either the rotary or manual techniques is distinct. Nevertheless, the rotary instruments presented less difficulty in modulating the joint torque in each articulation, thus, presenting a greater uniformity of movements. Concerning the ergonomic aspect, alternating rotary and manual instrumentation for root canal preparation might contribute to a decrease in the occurrence of WMSD. In other words, alternating the use of the manual and rotary techniques would produce a better balance in the activation of the muscles, because the motor strategies are different. Different motor strategies can be found when there is variability of the tasks ${ }^{12}$ and when pain is present ${ }^{11}$, and they can be a mechanism of protection against WMSD. However, further studies should be carried out to investigate this hypothesis.

\section{CONCLUSIONS}

Based on the methodology used and the results obtained, it can be concluded that:

The angular displacement of the wrist and elbow during root canal preparation using rotary and manual instruments were classified as low risk in terms of the occurrence of WMSD;

There were no significant differences between the kinetic records for the two instrumentation techniques;

A higher level of muscular activity was verified in the majority of the muscles assessed (extensor carpi radialis, flexor carpi radialis, brachioradialis and triceps brachii), although the motor strategy was shown to be distinct for each of the two techniques tested.

\section{REFERENCES}

1- Akesson I, Lundborg G, Horstmann V, Skerfving S. Neuropathy in female dental personnel exposed to high frequency vibrations. Occup Environ Med. 1995;52:116-23.

2- Alexopoulos EC, Stathi IC, Charizani F. Prevalence of musculoskeletal disorders in dentists. BMC Musculoskelet Disord. 2004;5:1-16.

3- Andersson GBJ. Epidemiologic features of chronic low-back pain. Lancet. 1999;354(9178):581-5.

4- Bramson JB, Smith S, Romagnoli G. Evaluating dental office ergonomic. Risk factors and hazards. J Am Dent Assoc. 1998;129:174-83.

5- De Leva P. Joint center longitudinal positions computed from a selected subset of Chandler's data. J Biomech. 1996;29:1231-3. 6- Djerassi E. Some problems of the occupational diseases of dentists. Int Dent J. 1971;21(2):252-9.

7- Gluskin AH, Brown DC, Buchanan LS. A reconstructed computerized tomographic comparison of $\mathrm{Ni}$ - $\mathrm{Ti}$ rotary $\mathrm{GT}$ files versus traditional instruments in canals shaped by novice operators. Int Endod J. 2001;34:476-84.

8- Guay AH. Commentary: ergonomically related disorders in dental practice. J Am Dent Assoc. 1998;129:184-6.

9- Kendall FP, McCreary EK, Provance PG, McIntyre M. Muscles: testing and function. $5^{\text {th }}$ ed. Baltimore MD: Lippincott Williams \& Wilkins; 2005.

10- Larsson R, Oberg PA, Larsson SE. Changes of trapezius muscle blood flow and electromyography in chronic neck pain due to trapezius myalgia. Pain. 1999;79:45-50.

11- Madeleine P, Voigt M, Mathiassen SE. The size of cycle-to-cycle variability in biomechanical exposure among butchers performing a standardised cutting task. Ergonomics. 2008;51:1078-95

12- Mathiassen SE, Möller T, Forsman M. Variability in mechanical exposure within and between individuals performing a highly constrained industrial work task. Ergonomics. 2003;46:800-24. 13- Morse TF, Michalak-Turcotte C, Atwood-Sanders M, Warren $\mathrm{N}$, Peterson DR, Bruneau $\mathrm{H}$, et al. A pilot study of hand and arm musculoskeletal disorders in dental hygiene students. J Dent Hyg. 2003;77:173-9.

14- Ozawa T, Nagumo S, Kikuchi K, Yamazaki Y, Nakamura J. A survey of the symptoms in dentists' hands and forearms during or after dental practice. Tsu Univ Dent J. 1998(3);25:35-41.

15- Ozawa T, Nakano M, Sugimura H, Kurayama H, Tahata K, Nakamura J, et al. Effects of endodontic instrument handle diameter on electromyographic activity of forearm and hand muscles. Int Endod J. 2001;34:100-6. 
16- Parsell DE, Weber MD, Anderson BC, Cobb-Junior GW Jr. Evaluation of ergonomic dental stools through clinical simulation. Gen Dent. 2000;48:440-4.

17- Pasternak-Júnior B, Sousa-Neto MD, Silva RG. Canal transportation and centring ability of RaCe rotary instruments. Int Endod J. 2009;42:499-506.

18- Regis-Filho GI. Epidemiologic and ergonomic aspects of musculoskeletal disorders in dentists. Rev APCD. 1997;51:469-75. 19- Sande de Souza LAP, Dionisio VC, Lerena MAM, Marconi NF, Almeida GL. The linear co-variance between joint muscle torques is not a generalized principle. J Electromyogr Kinesiol. 2007; 19:171-9.

20- Schneider K, Zernicke RF, Schmidt RA, Hart TJ. Changes in limb dynamics during the practice of rapid arm movements. J Biomech. 1989;22:805-17

21- Short JA, Morgan LA, Baumgartner JC. A comparison of canal centering ability of four instrumentation techniques. J Endod. $1997 ; 23: 503-7$
22- Simmer-Beck M, Bray KK, Bramson B, Glaros A, Weeks J. Comparison of muscle activity associated with structural differences in dental hygiene mirrors. J Dent Hyg. 2006;80:8.

23- Szymańska J. Disordes of the musculoskeletal system among dentists from the aspect of ergonomics and prophylaxis. Ann Agric Environ Med. 2002;9:169-73.

24- Taşdemir $\mathrm{T}$, Aydemir $\mathrm{H}$, Inan $U$, Unal O. Canal preparation with Hero 642 rotary $\mathrm{Ni}-\mathrm{Ti}$ instruments compared with stainless steel hand $\mathrm{K}$-file assessed using computed tomography. Int Endod J. 2005;38:402-8.

25- Treaster D, Marras WS, Burr D, Sheedy JE, Hart D. Myofascial trigger point development from visual and postural stressors during computer work. J Electromyogr Kinesiol. 2006;16:115-24. 26- Wheeler AH, Goolkasian P, Gretz SS. Botulinum toxin A for the treatment of chronic neck pain. Pain. 2001;94:255-60.

27- Young GR, Parashos $\mathrm{P}$, Messer $\mathrm{HH}$. The principles of techniques for cleaning root canals. Aust Dent J. 2007;52:52-63. 